\title{
Cateterismo dei vasi venosi centrali: nec tecum, nec sine te beate vivere possumus
}

\author{
Luisa Berardinelli
}

\author{
Direttore della U.O. di Chirurgia Vascolare e Trapianti di Rene, \\ Ospedale Maggiore Policlinico di Milano e \\ Cattedra di Chirurgia dei Trapianti d'Organo ed Organi Sostitutivi, \\ Università di Milano, Milano
}

\section{Il razionale}

$\mathbf{L}$ a creazione e il mantenimento dell'accesso vascolare costituisce tuttora il vero "tallone di Achille" del trattamento sostitutivo nell'insufficienza renale terminale. Se all'inizio degli anni '60 il trattamento emodialitico d'urgenza veniva effettuato con l'applicazione degli shunts di Scribner-Quinton, di Ramirez, di Thomas o di Allen Brown, negli ultimi venti anni il cateterismo percutaneo dei grossi vasi venosi centrali (CVC), in particolare degli arti superiori, ha in pratica soppiantato gli shunts esterni, e, in certi Centri, addirittura sostituito le fistole arterovenose con i vasi nativi. Attualmente, secondo il DOPPS 2000 (Dialysis Outcomes and Practice Patterns Study) (1), su un campionamento random di 10,332 pazienti statunitensi, il $59 \%$ di essi inizia l'emodialisi con un CVC, il $25 \%$ con un accesso protesico e solo il $15 \%$ con una fistola arterovenosa, mentre in Europa le corrispondenti percentuali sono, rispettivamente di $30 \%, 3 \%$ e $67 \%$.

Il riferimento tardivo del paziente allo specialista nefrologo, la rapidità di in- serzione di tali accessi venosi, l'incidenza relativamente contenuta di complicanze maggiori collegate alla metodica, la possibilità di ottenere immediatamente flussi dialitici adeguati, senza conseguenze emodinamiche, ma anche un modello di "gestione autarchica" da parte del nefrologo, che preferisce non ricorrere alla collaborazione del chirurgo vascolare e la scarsa conoscenza delle conseguenze endovasali di un cateterismo dei grossi vasi centrali, anche di breve durata, sono tutti fattori che hanno concorso alla diffusione, attualmente indiscriminata, di tale metodica.

Altri fattori, che vengono chiamati frequentemente in causa per giustificare la diffusione del cateterismo venoso centrale, sono costituiti dall'esaurimento dei vasi superficiali, dall'aumento della anzianità anagrafica e dialitica dei pazienti uremici, dall'aumentata incidenza di diabete e patologie complesse nella popolazione dialitica, che rendono difficoltoso e talora impossibile l'accesso vascolare chirurgico. Non va infine sottostimata la preferenza che alcuni pazienti, non sufficientemente informati dal medico sulle possibili future complicanze, ac- cordano al trattamento dialitico con catetere venoso centrale permanente. Tale metodica infatti prevede un accesso non traumatico al sistema vascolare, mentre le fistole arterovenose derivano il flusso ematico mediante puntura con aghi di grosso calibro.

\section{Come si identifica una stenosi/ trombosi dei vasi venosi centrali}

La presenza di una stenosi dei vasi venosi centrali può essere sospettata per la presenza di un circolo collaterale sull'emitorace e sull'omero corrispondente, ma può anche rimanere del tutto asintomatica finché non viene costruita una fistola arterovenosa (FAV) dallo stesso lato. L'esame dopplersonografico fornisce solo segni indiretti di stenosi, ma nella nostra esperienza l'unico test affidabile al $100 \%$ è rappresentato dalla flebografia, allargata all'emitorace corrispondente e praticata con l'arto superiore in abduzione. Infatti, se l'arto viene mantenuto addotto, si possono evidenziare stenosi, che in realtà sono solo funzionali. L'edema dell'arto si verifica in tal caso entro pochi giorni $o$ addirittura entro poche ore dall'isti- 
tuzione della FAV e, se la stenosi non viene trattata, finisce per provocare ulcere e vere e proprie gangrene venose, che possono essere risolte da manovre di dilatazione percutanea e posizionamento di stent dal radiologo interventista. Dopo la dilatazione, il paziente deve essere sottoposto a controlli periodici, poiché la stenosi può recidivare, con necessità di nuovi interventi di radiologia interventistica.

Se il radiologo non riesce a risolvere la stenosi, e insorgono gangrene venose, non rimane altra soluzione che lo smantellamento della FAV stessa.

\section{Perché "far macchina indietro" sul trattamento dialitico con CVC}

Significativamente inferiore appare la sopravvivenza attuariale di un accesso vascolare, eseguito con i vasi nativi o con protesi interposta, se il paziente è stato sottoposto a un cateterismo temporaneo dei grossi vasi centrali. Secondo la segnalazione del DOPPS (1), le fistole arterovenose costruite con vasi nativi nei pazienti statunitensi, che non avevano in precedenza posizionato un CVC, presentano a un anno il $75 \%$ di sopravvivenza; il tasso di sopravvivenza, rilevato allo stesso periodo, crollava drammaticamente al $40 \%$ nei pazienti, che avevano iniziato la dialisi con un CVC.

Inoltre, se appare elevata la percentuale del $30 \%$ di pazienti, che in Europa iniziano l'emodialisi con un CVC e ancor più il 59\% negli USA, in effetti una percentuale assai più elevata, e difficilmente quantificabile, di questi pazienti viene sottoposto almeno una volta durante la propria vita di uremico per periodi variabili a un cateterismo dei grossi vasi centrali a causa in genere di un malfunzionamento della fistola arterovenosa (2).

Il CVC può determinare anche dopo sole tre settimane una stenosi, o addirittura una trombosi dei grossi vasi centrali, soprattutto se introdotta nella vena succlavia (3). L'uso diffuso delle vene giugulari diminuisce il rischio di stenosi $(4,5)$, ma non lo annulla.

Le vene giugulari, interna o esterna, possono tra l'altro essere usate per accessi vascolari insoliti (6), di durata ben maggiore di un qualunque $\mathrm{CVC}$, se non vi sono stati inseriti cateteri e se il drenaggio venoso centrale è pervio. Il problema della stenosi dei vasi centrali può essere risolto dal radiologo interventista, ma i costi sociali ed economici per i successivi controlli e nuovi periodici posizionamenti di stents diventano talora improponibili. A causa dei flussi ridotti, il CVC richiede tra l'altro periodi di dialisi più lunghi rispetto alla FAV.

Il CVC è soggetto a frequenti trombosi, che richiedono l'impegno di tempo, strutture e costosi farmaci per la risoluzione.

Altro notevole problema del CVC è costituito dal rischio di infezione, che in uno studio recente raggiunge il $40 \%$ in un periodo di 9 mesi (7). Inoltre, se un catetere è infetto, non può essere costruita una fistola arterovenosa con vasi nativi, né tanto meno con protesi vascolari, per il rischio elevatissimo di arterite settica e/o sepsi.

\section{Conclusioni}

Nel contesto del trattamento dialitico, fra le decisioni "critiche", quella che riguarda l'uso del cateterismo dei vasi venosi centrali appare oggi sicuramente la più importante nel condizionare l'aspettativa di vita del paziente uremico.

Ancora una volta va ribadito il concetto che la fistola arterovenosa rimane tuttora il "cavallo di battaglia" più affidabile per l'emodialisi e che la fantasia del chirurgo può concretarsi nella confezione di accessi vascolari anche insoliti (6), soltanto se esiste un idoneo drenaggio venoso.

La presenza di stenosi o trombosi della vena succlavia, del tronco anonimo o addirittura della vena cava superiore, impedisce la confezione di una fistola arterovenosa sugli arti superiori $\mathrm{o}$ addirittura può richiedere lo smantellamento di una fistola ben funzionante, a causa di un edema dell'arto omolaterale, se il radiologo interventista non riesce a trattarla.

Il nefrologo deve imparare a diventare estremamente selettivo nel decidere il posizionamento di un CVC, per assicurare una lunga vita all'accesso vascolare permanente, e di conseguenza allo stesso paziente in emodialisi, riservandolo solo a casi veramente eccezionali.

Accorgimenti utili a prevenire la necessità di un catetere venoso centrale sono:

- Una conservazione "maniacale" del patrimonio vascolare superficiale del paziente uremico.

- Evitare l'arteriografia.

- Coinvolgere precocemente il chirurgo vascolare per la confezione degli accessi.

- Anticipare la confezione della FAV con vasi nativi, per permetterne uno sviluppo sufficiente.

- Rivalutare l'uso della dialisi peritoneale per trattare l'urgenza e nel periodo di maturazione della FAV.

- Nel caso sia assolutamente necessaria un'emodialisi d'urgenza, privilegiare il cateterismo della vena giugulare interna destra, associato a un minor rischio di complicanze, o della vena femorale.

- Il trattamento emodialitico con catetere temporaneo non dovrebbe mai superare la durata di tre settimane.

- L'età assai giovane o molto avanzata del paziente, osservando particolari cautele, non controindicano la confezione di accessi vascolari chirurgici.

- Il paziente deve sapere che il cateterismo permanente dei grossi vasi rappresenta l' "ultima spiaggia", in quanto preclude la possibilità di costruire un accesso chirurgico per un tempo assai lungo, spesso per sempre, a causa della trombosi dei vasi centrali, che esso infine determina.

\section{luisa.berardinelli@unimi.it}




\section{BIBLIOGRAFIA}

1. National Kidney Foundation. K/DOQI Clinical Practice Guidelines for Vascular Access, 2000. Am J kidney Dis 2001; 37 (Suppl 1): S137-81.

2. Kumwenda MJ, Wright FK and Haybittle DJ . Survey of permanent central venous catheters for haemodialysis in the UK. Nephrol Dial Transplant 1996; 11: 830-2.

3. Cimochowsky GE, Worley E, Rutherford WE, Sartain J, Blodin J, Harter H. Superiority of the internal jugular over the subclavian access for temporary dialysis. Nephron 1990; 54: 154.

4. Uldall PR. Subclavian cannulation is no longer necessary or justified in patients with end-stage renal failure. Semin Dial 1994; 7: 161-4.

5. Konner K. Subclavian haemodialysis access: is still justified in 1995? Nephrol Dial Transplant 1995; 1988-9.

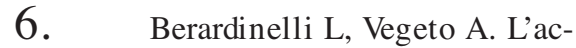
cesso vascolare per la dialisi extracorporea. Monografia con videocassetta. Torino, UTET, Periodici Scientifici, 1993.

7. Marr KA, Sexton DJ, Colon PJ, et al. Catheter-related bacteremia and outcome of attemped catheter salvage in patients undergoing hemodialysis. Ann Intern Med 1997; 127: 275-80. 